\title{
Research on Image Acquisition and Recognition for Stored Grain Pests
}

\author{
Defa Wang ${ }^{1}$, Huiling Zhou ${ }^{1, *}$, Haiying Yang ${ }^{1}$, Yufeng Shen ${ }^{1}$, Yang Cao ${ }^{2}$ and Huiyi Zhao ${ }^{2}$ \\ ${ }^{1}$ Beijing University of Posts and Telecommunications, China \\ ${ }^{2}$ Academy of State Administration of Grain, China
}

\begin{abstract}
Manual ways of recognizing stored grain pests which are trapped is very time-consuming. In this study, an image dataset of 9 species of pests was built up by finding the MSERs (Maximally Stable Extremal Regions), through a trap of stored grain pests combined with a real-time imaging device. On this basis, the localization and recognition of stored grain pests were achieved. The experimental results on 3600 images showed that by the combination of shape features and color features, the average $F_{1}$ score was about 0.947 by selecting the appropriate parameters of SVM (Support Vector Machines) classifier.
\end{abstract}

Keywords- stored grain pests; image dataset; MSERs; color features; shape features

\section{INTRODUCTION}

In the area of recognizing stored grain pests, various methods based on machine vision had been proposed. In 1998, Zayas was first reported using color features to detect the Rhizopertha Dominica (RD) beetles in bulk wheat samples and obtained a recognition rate higher than 90\% [1]. By introducing color, texture and shape features to a SVM (Support Vector Machine) classifier, YAO [2] et al got a $97.5 \%$ average accurate rate for four species of Lepidoptera rice pests which were trapped by a rice light-trap in paddy field.

By putting the siftage of grain sample on the conveyor belt, Zhang et al [3] [4] obtained a recognition rate of $75.3 \%$ based on 21 overall shape features for 15 species of stored grain pests. On this basis, they extracted 7 local shape features and reached a recognition rate of $94.8 \%$.

By taking pictures for the trapped pests, Zhen [5] extracted color features based on statistical characteristics of RGB histogram and texture features based on gray level run length matrix to enhance the ability to identify stored grain pests based on shape features and a recognition rate of $94 \%$ was obtained; Zhang [6] [7] extracted statistical, texture and shape features to identify the stored grain pests and reached a recognition rate of $98 \%$. But they just took three species of stored grain pests into consideration, namely, Sitophilus Oryzae Linne (SOL), Tribolium Confusum (TC), Oryzaephilus Surinamensis Linne (OSL).

Therefore, for the real-time monitoring and recognition of stored grain pests in the grain warehouse, this paper used a trap and online monitoring device developed by our research group. First, we simulated the process of trapping stored grain pests in laboratory and took pictures of stored grain pests which were trapped. Second, an image dataset including 9 species of stored grain pest was built based on the region detection method. Finally, three experiments were carried out and confirmed that shape, color features are very effective for the classification of those 9 species of stored grain pests.

\section{MAterials AND Methods}

\section{A. Image Acquisition}

Nine species of stored grain pests were used in this study, namely, OSL, Cryptolestes Turcicus (CT), Cryptolestes Ferrugineus (CF), Tribolium Castaneum Herbst (TCH), SOL, Sitophilus Zeamais (SZ), RD, Attagenus Unicolor Japonicus (AUJ), Lasioderma Serricorne (LS). The samples were provided by National Engineering Laboratory for Grain Storage and Logistics.

The trap and online monitoring device for stored grain pests is shown in figure I (b). Figure I(a) shows the flow chart of the image acquisition system. At first, stored grain pests are trapped and images are taken by a CCD camera (MV-GE500C$\mathrm{T}$ ) with a prime lens of $8 \mathrm{~mm}$ focal length (M0814-MP2) above the trap. Then, those images are transmitted to a personal computer by the data transmission terminal. Finally, images are uploaded to the server where image processing and recognition are carried out.
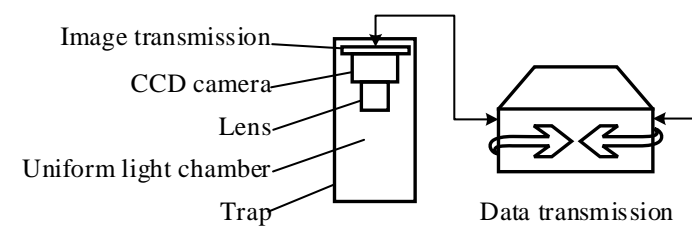

(a)

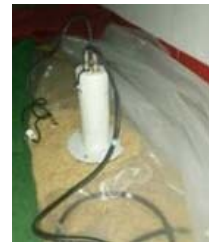

(b)

FIGURE I(a) THE FLOW CHART OF THE IMAGE ACQUISITION SYSTEM; (b) THE TRAP AND ONLINE MONITORING DEVICE 


\section{B. Localization of Stored Grain Pests}

In terms of the fact that images acquired by the CCD camera usually contains several pests, our first step was to obtain images which contained only a single pest for the convenience of recognition. A region detection method called MSERs was used to detect the ROI (Region of Interest) and region merging based on the overlap ratio of the ROI was performed. The result is shown in figure II(a). Then the original image was cropped according to the minimum enclosing rectangle of these ROI, so we got a series of images shown in figure II (b) to figure II (m).

A small piece of images obtained by the above method contained multiple pests or didn't contain a complete pest, as shown in figure II(k) to figure II(m). In this study, we didn't take those images into consideration. For an image containing a single complete pest, the image was labeled as different kinds of pests by manual ways. Then the image dataset for nine

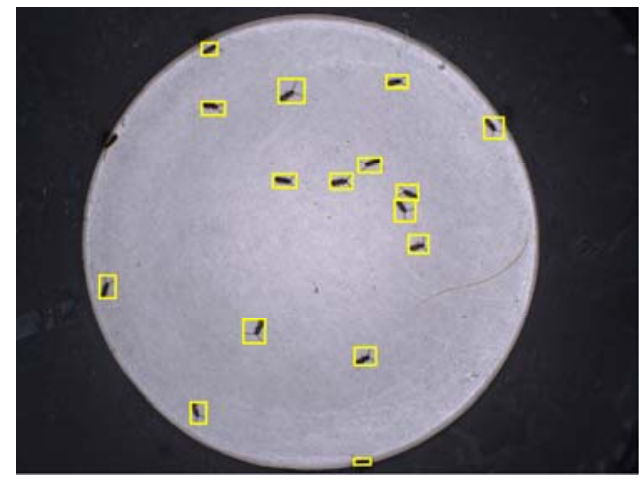

(a)

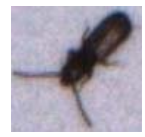

(b)

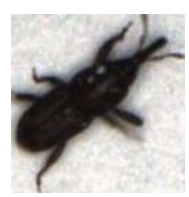

(e)

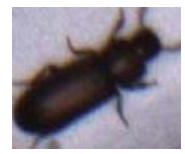

(h)

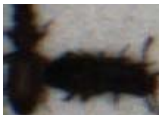

(k)

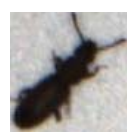

(c)

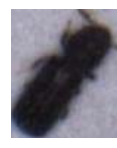

(f)

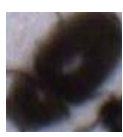

(i)

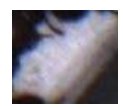

(l)

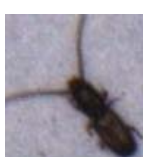

(d)

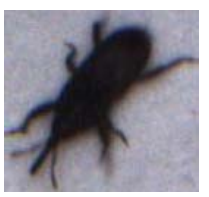

(g)

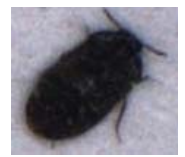

(j)

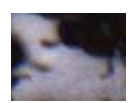

(m)
FIGURE II. (a) THE BOUNDING BOX OF THE MERGED ROI; (b) CT; (c) OSL; (d) CF; (e) SOL; (f) RD; (g) SZ; (h) TCH; (i) LS; (j) AUJ; (k) MERGED ROI Which Contains Multiple Pests; (l) MERGED ROI WHICH DON'T CONTAIN COMPLETE PEST; (m) MERGED ROI WHICH DON'T CONTAIN COMPLETE PEST. species (400 images for each pest) of pests was obtained. Figure II(b) to figure II(j) shows the image samples for those pests.

\section{Features Extraction}

Based on the image dataset obtained above, a fixed threshold segmentation method called OTSU [8] was used and binary image was obtained by area filter, hole filling method. On the basis of binary image, shape and color features of pests were extracted.

1) Shape features. For those nine species of pests, it is obvious that some pests are different from other pests in terms of shape as shown in figure $\mathrm{II}(\mathrm{b})$ to figure $\mathrm{II}(\mathrm{j})$, e.g. : OSL is more slender than CT and CF; AUJ and LS is bigger than CT and $\mathrm{CF}$. Therefore, area (A), perimeter (PE), equivalent diameter (D), major axis (MA), minor axis (MI) and eccentricity (E) of the binary image were extracted. The area was estimated by the number of pixels of the binary image; the perimeter was estimated by the pixels on the edge of the binary image and the equivalent diameter was obtained through a circle which had the same area of the target area.

In addition, major axis (MA), minor axis $(M I)$ and eccentricity $(E)$ were obtained through the ellipse fitting parameters of the target area. The principle of ellipse fitting was keeping the second order moments of original target area and the ellipse the same [9]. The detail is as follows:

Suppose the binary image is $I(x, y)$, then, we can define the zero, first order moments as follows:

$$
\begin{gathered}
m_{00}=\sum I(x, y) \\
m_{10}=\sum x \times I(x, y) \\
m_{01}=\sum y \times I(x, y)
\end{gathered}
$$

Where $m_{00}$ represents the area.

So if we define the center of the region as $\left(x_{\mathrm{c}}, y_{\mathrm{c}}\right)$, it can be calculated as follows:

$$
\begin{aligned}
& x_{\mathrm{c}}=\frac{m_{10}}{m_{00}} \\
& y_{\mathrm{c}}=\frac{m_{01}}{m_{00}}
\end{aligned}
$$

Then, we can define the matrix of the centered second order moments as follows: 


$$
\begin{gathered}
\boldsymbol{U}_{2}=\left[\begin{array}{ll}
u_{20} & u_{11} \\
u_{11} & u_{02}
\end{array}\right] \\
u_{20}=\sum\left(x-x_{c}\right)^{2} \times I(x, y) \\
u_{02}=\sum\left(y-y_{c}\right)^{2} \times I(x, y) \\
u_{11}=\sum\left(x-x_{c}\right) \times\left(y-y_{c}\right) \times I(x, y)
\end{gathered}
$$

From the matrix $\boldsymbol{U}_{2}$ above, the eigenvalue, namely, $\lambda_{1}$ and $\lambda_{2}$ can be derived. Then the MA, MI, E can be defined as follows:

$$
\begin{gathered}
M A=\sqrt{\frac{\lambda_{1}}{m_{00}}} \\
M I=\sqrt{\frac{\lambda_{2}}{m_{00}}} \\
E=\frac{\sqrt{M A^{2}-M I^{2}}}{M A}
\end{gathered}
$$

2) Color features. Although we could distinguish OSL from SOL easily only by shape features, it was still hard to make a distinction between AUJ and LS. Besides, the size of pest changes as time went on. From the figure II(i) to figure II(j), big differences between AUJ and LS can be found because AUJ is darker than LS. Therefore, eighteen color features based on the region RGB color histogram were extracted. The detail of the color features extraction was as follows: from the binary image and the original image, three histograms of the target area could be calculated (one for each channel of the color); then, using six statistics to describe the histogram, namely, mean, standard deviation, smoothness, three order moment, consistency and entropy [2], so we could get the eighteen color features species of pests.

\section{Image Classification}

Nine species of pests (400 images for each species) were taken into consideration. In the classification experiment, in order to prevent over fitting, the technique of five-fold crossvalidation was used to obtain the recognition rate. It means the images of each pests were divided into five mutually disjoint subsets (each subset contains 80 images). During the training time, each subset out of the five subsets was extracted as a validation set and the remaining four subsets would be used to train the classifier, so we could evaluate the recognition rate on the validation set.

1) SVM. First proposed by Vapnik et al, SVM is a supervised machine learning method which is aimed at finding the optimum decision separating hyperplane. The core of this algorithm is mainly based on maximizing the minimum Euclidean distance between the sample and the decision separating hyperplane directly. When it comes to linearly nonseparable problem, there are two common ways to solve this problem: (1) adding a penalty term and introducing a super parameter $C$ to balance the structural risk and empirical risk; (2) mapping the features to higher dimensional space by a kernel function, then it can be linearly separable in high dimensional space. In this paper, in order to improve the recognition rate, the experiments showed that the quartic polynomial kernel function with a penalty factor $C=2$ could achieve the best results.

But the traditional SVM is a classifier to deal with binary classification problem, so we transformed multi classification problem into several binary classification problems. It means, for very possible pair of pests, a SVM classifier was trained and all those classifiers were combined together by using the voting method to get the final classification.

2) Image classification experiments. In order to verify that shape, color features were effective for the classification of nine species of pests, three classification experiments were designed delicately. The first experiments was based on six shape features and the results would be discussed in Section 3.1; experiment two used eighteen color features for the classification and the result would be discussed in Section 3.2; experiment three combined shape and color features and the results would be discussed in section 3.3. Besides, super parameters mentioned in section 2.3.1 were adopted in the three experiments.

\section{TABLE I. CONFUSION MATRIX}

\begin{tabular}{|c|c|c|}
\hline \multirow{2}{*}{$\begin{array}{c}\text { True } \\
\text { labels }\end{array}$} & \multicolumn{2}{|c|}{ Predict labels } \\
\cline { 2 - 3 } Positive & Positive & Negative \\
\hline Negative & $F P$ & $F N$ \\
\hline
\end{tabular}

The results of the experiments were evaluated based on the precision rate $(P)$, recall rate $(R)$ and $F_{1}$ score, in which the average recall rate was regarded as the recognition rate. If Table I is the confusion matrix of the classification result, then $P, R, F_{1}$ can be defined as follows:

$$
\begin{aligned}
& P=\frac{T P}{T P+F P} \\
& R=\frac{T P}{T P+F N}
\end{aligned}
$$




$$
F_{1}=\frac{2 \times P \times R}{P+R}
$$

\section{RESUlts AND DisCUSSION}

\section{A. Classification Results Based on Shape Features}

Table II shows the confusion matrix, precision rate, recall rate and $F_{1}$ score of classification based on shape features. From the row of the recall rate, it can be seen that CT (77.3\%), SZ (64.0\%), AUJ (85.5\%), SOL (84.3\%) and CF (54.5\%) get a relatively low recall rate. Connected to the confusion matrix and, it is obvious that there are too many misclassifications (246 errors) between the two species CT and CF. It is the same for the pairs of SOL and SZ (176 errors), AUJ and LS (51 errors). This means it is difficult to separate between those pairs if only shape features are used. RD received a relatively high precision rate $(96.7 \%)$, recall rate $(96.0 \%)$ and $F_{1}$ score
(0.964), which means shape features are effective to distinguish $\mathrm{RD}$ from other pests. The overall average $F_{1}$ score is 0.824 .

\section{B. Classification Results Based on Color Features}

Table III shows the confusion matrix, precision rate, recall rate and $F_{1}$ score of classification based on color features. From the row of the recall rate, it is obvious that SZ (96.5\%), AUJ (96.3\%), SOL (98.8\%) and CF $(73.5 \%)$ get a relatively high recall rate compared to the classification results based on shape features. From the confusion matrix, classifier based on the color features can't differentiate between CT and CF, so a relatively low $F_{1}$ score is given to those species of pests. From the row of the $F_{1}$ score, the classification performance has been greatly improved, especially on LS (0.978) and AUJ (0.971), SZ (0.966) and SOL (0.983). In addition, a progress has been made on other species of pests, just as TCH (0.945) and OSL (0.971). The overall average $F_{1}$ score is about $92.7 \%$.

TABLE II. CONFUSION MATRIX, PRECISION RATE, RECALL RATE AND F1 SCORE BASED ON SHAPE FEATURES

\begin{tabular}{|c|c|c|c|c|c|c|c|c|c|}
\hline \multirow{2}{*}{$\begin{array}{c}\text { True } \\
\text { labels }\end{array}$} & \multicolumn{10}{|c|}{ Predicted labels } \\
\cline { 2 - 11 } & $\boldsymbol{C T}$ & $\boldsymbol{L S}$ & $\boldsymbol{S Z}$ & $\boldsymbol{A U \boldsymbol { U }}$ & $\boldsymbol{S O L}$ & $\boldsymbol{R D}$ & $\boldsymbol{T C H}$ & $\boldsymbol{C F}$ & $\boldsymbol{O S L}$ \\
\hline $\boldsymbol{C T}$ & 309 & 0 & 0 & 0 & 0 & 1 & 0 & 84 & 6 \\
\hline $\boldsymbol{L} \boldsymbol{S}$ & 0 & 389 & 2 & 7 & 0 & 1 & 0 & 0 & 1 \\
\hline $\boldsymbol{S Z}$ & 0 & 2 & 256 & 0 & 122 & 3 & 16 & 0 & 1 \\
\hline $\boldsymbol{A U \boldsymbol { U }}$ & 1 & 44 & 2 & 342 & 1 & 2 & 8 & 0 & 0 \\
\hline $\boldsymbol{S O L}$ & 1 & 0 & 54 & 0 & 337 & 1 & 7 & 0 & 0 \\
\hline $\boldsymbol{R D}$ & 0 & 2 & 1 & 1 & 0 & 384 & 0 & 0 & 12 \\
\hline $\boldsymbol{T C H}$ & 0 & 4 & 14 & 12 & 13 & 1 & 355 & 1 & 0 \\
\hline $\boldsymbol{C F}$ & 162 & 0 & 0 & 0 & 0 & 0 & 0 & 218 & 20 \\
\hline $\boldsymbol{O S L}$ & 8 & 1 & 1 & 1 & 0 & 4 & 1 & 0 & 384 \\
\hline Precision & $64.2 \%$ & $88.0 \%$ & $77.6 \%$ & $94.2 \%$ & $71.2 \%$ & $96.7 \%$ & $91.7 \%$ & $71.9 \%$ & $90.6 \%$ \\
\hline Recall & $77.3 \%$ & $97.3 \%$ & $64.0 \%$ & $85.5 \%$ & $84.3 \%$ & $96.0 \%$ & $88.8 \%$ & $54.5 \%$ & $96.0 \%$ \\
\hline $\boldsymbol{F}_{\mathbf{1}}$ & 0.701 & 0.924 & 0.701 & 0.896 & 0.772 & 0.964 & 0.902 & 0.620 & 0.932 \\
\hline
\end{tabular}

TABLE III. CONFUSION MATRIX, PRECISION RATE, RECALL RATE AND $F_{1}$ SCORE BASED ON COLOR FEATURES

\begin{tabular}{|c|c|c|c|c|c|c|c|c|c|}
\hline \multirow{2}{*}{$\begin{array}{c}\text { True } \\
\text { labels }\end{array}$} & \multicolumn{10}{|c|}{ Predicted labels } \\
\cline { 2 - 10 } & $\boldsymbol{C T}$ & $\boldsymbol{L S}$ & $\boldsymbol{S Z}$ & $\boldsymbol{A U J}$ & $\boldsymbol{S O L}$ & $\boldsymbol{R D}$ & $\boldsymbol{T C H}$ & $\boldsymbol{C F}$ & $\boldsymbol{O S L}$ \\
\hline $\boldsymbol{C T}$ & 325 & 1 & 0 & 0 & 0 & 0 & 8 & 65 & 1 \\
\hline $\boldsymbol{L} \boldsymbol{S}$ & 1 & 393 & 0 & 1 & 0 & 0 & 1 & 3 & 1 \\
\hline $\boldsymbol{S Z}$ & 0 & 1 & 386 & 4 & 1 & 5 & 3 & 0 & 0 \\
\hline $\boldsymbol{A U \boldsymbol { U }}$ & 0 & 0 & 8 & 385 & 1 & 6 & 0 & 0 & 0 \\
\hline $\boldsymbol{S O L}$ & 0 & 0 & 0 & 0 & 395 & 0 & 0 & 0 & 5 \\
\hline $\boldsymbol{R D}$ & 0 & 0 & 2 & 3 & 0 & 395 & 0 & 0 & 0 \\
\hline $\boldsymbol{T C H}$ & 4 & 4 & 3 & 0 & 0 & 2 & 377 & 8 & 2 \\
\hline $\boldsymbol{C F}$ & 95 & 3 & 0 & 0 & 0 & 0 & 6 & 294 & 2 \\
\hline $\boldsymbol{O S L}$ & 0 & 2 & 0 & 0 & 7 & 0 & 3 & 0 & 388 \\
\hline Precision & $76.5 \%$ & $97.3 \%$ & $96.7 \%$ & $98.0 \%$ & $97.8 \%$ & $96.8 \%$ & $94.7 \%$ & $79.5 \%$ & $97.2 \%$ \\
\hline Recall & $81.3 \%$ & $98.3 \%$ & $96.5 \%$ & $96.3 \%$ & $98.8 \%$ & $98.8 \%$ & $94.3 \%$ & $73.5 \%$ & $97.0 \%$ \\
\hline $\boldsymbol{F}_{\mathbf{1}}$ & 0.788 & 0.978 & 0.966 & 0.971 & 0.983 & 0.978 & 0.945 & 0.764 & 0.971 \\
\hline
\end{tabular}

\section{Classification Results Based On Shape and Color Features}

Table IV shows the confusion matrix, precision rate, recall rate and $F_{1}$ score of classification based on shape and color features. The misclassifications between LS and AUJ, SOL and SZ have been solved effectively compared to the result based on shape features which has been discussed in section 3.1. Besides, compared to the result based on color features which has been discussed in 3.2, the misclassifications between CT and TCH, AUJ and SZ, SOL and OSL, TCH and CF have also been solved effectively. From the row of $F_{1}$ score, LS (0.995), SZ (0.980), AUJ (0.989), SOL (0.990), RD (0.994), TCH (0.986), OSL (0.990) get a score higher than 0.98 , which means that those seven species of pests can be identified effectively. Meanwhile, although the $F_{1}$ score of CT and CF has been improved, a large number of samples remain misclassified. The final average $F_{1}$ score is 0.947 . 
TABLE IV. CONFUSION MATRIX, PRECISION RATE, RECALL RATE AND $F_{1}$ SCORE BASED ON SHAPE AND COLOR FEATURES

\begin{tabular}{|c|c|c|c|c|c|c|c|c|c|}
\hline \multirow{2}{*}{$\begin{array}{c}\text { True } \\
\text { labels }\end{array}$} & \multicolumn{10}{|c|}{ Predicted labels } \\
\cline { 2 - 10 } & $\boldsymbol{C T}$ & $\boldsymbol{L S}$ & $\boldsymbol{S Z}$ & $\boldsymbol{A U J}$ & $\boldsymbol{S O L}$ & $\boldsymbol{R D}$ & $\boldsymbol{T C H}$ & $\boldsymbol{C F}$ & $\boldsymbol{O S L}$ \\
\hline $\boldsymbol{C T}$ & 339 & 0 & 0 & 0 & 0 & 0 & 0 & 59 & 2 \\
\hline $\boldsymbol{L} \boldsymbol{S}$ & 0 & 398 & 1 & 0 & 0 & 0 & 1 & 0 & 0 \\
\hline $\boldsymbol{S Z}$ & 0 & 1 & 395 & 0 & 1 & 0 & 3 & 0 & 0 \\
\hline $\boldsymbol{A U \boldsymbol { U }}$ & 0 & 0 & 2 & 393 & 3 & 2 & 0 & 0 & 0 \\
\hline $\boldsymbol{S O L}$ & 0 & 0 & 1 & 1 & 398 & 0 & 0 & 0 & 0 \\
\hline $\boldsymbol{R} \boldsymbol{D}$ & 0 & 0 & 1 & 0 & 0 & 399 & 0 & 0 & 0 \\
\hline $\boldsymbol{T C H}$ & 0 & 0 & 5 & 1 & 0 & 0 & 393 & 1 & 0 \\
\hline $\boldsymbol{C F}$ & 98 & 0 & 0 & 0 & 0 & 1 & 0 & 301 & 0 \\
\hline $\boldsymbol{O S L}$ & 0 & 1 & 1 & 0 & 2 & 1 & 0 & 1 & 394 \\
\hline Precision & $77.6 \%$ & $99.5 \%$ & $97.3 \%$ & $99.5 \%$ & $98.5 \%$ & $99.0 \%$ & $99.0 \%$ & $83.1 \%$ & $99.5 \%$ \\
\hline Recall & $84.8 \%$ & $99.5 \%$ & $98.8 \%$ & $98.3 \%$ & $99.5 \%$ & $99.8 \%$ & $98.3 \%$ & $75.3 \%$ & $98.5 \%$ \\
\hline $\boldsymbol{F}_{\mathbf{1}}$ & 0.810 & 0.995 & 0.980 & 0.989 & 0.990 & 0.994 & 0.986 & 0.790 & 0.990 \\
\hline
\end{tabular}

\section{CONCLUSION}

An image dataset including nine species of stored grain pests was built by using the trap and online monitoring device and the discrimination ability of shape, color features were discussed in details in this study.

The results showed a comparatively low $F_{1}$ score $(0.824)$ can be achieved by only using shape features and we could get a higher $F_{1}$ score $(0.927)$ based on color features. Finally, by the fusion of shape and color features, a $F_{1}$ score $(0.947)$ was achieved.

However, for the pests contacted with the inner-wall of the trap (figure I(a)), it is still difficult to extract those pests because the contrast between the inner-wall and pests is relatively low, so the trap need to be improved to provide high contrast. At the same time, for those images contain multiple pests or don't contain a complete pest, we should go further in the future. Besides, the misclassification between CT and CF is still quite serious and this is the problem needed to be solved urgently.

\section{ACKNOWLEDGMENT}

This work is partially supported by the Program of Introducing Talents of Discipline to Universities of China (B08004), and the 2015 Special Fund for Grain-scientific Research in the Public Interest (201513002).

\section{REFERENCES}

[1] Zayas, I. Y., and P. W. Flinn. "Detection of insects in bulk wheat samples with machine vision." Transactions of the Asae 41.3(1998):883888.

[2] Yao Qing, Lv Jun, Liu Qing-jie, Diao Guang-qiang, Yang Bao-jun et al. "An Insect Imaging System to Automate Rice Light-Trap Pest Identification." Journal of Integrative Agriculture 11.6(2012):978-985.

[3] Zhang Hong-tao, Li Fang, Hu Yuxia, Zhang Hengyuan. "Extraction of Local Morphological Features of Stored-grain Insects." Journal of Henan Agricultural Sciences 43.2(2014):84-87.

[4] Zhang Hongtao, Mao Hanping, Qiu Daoyin. " Features extraction for the stored-grain insect detection system based on image recognition technology." Transactions of the CSAE 25.2(2009):12053-12054.
[5] Zhen Tong, Fan Yanfeng. "Research of Grain Pests Detection and Classification Based on SVM." Computer Engineering 32.9(2006):167169.

[6] Zhang Hongmei, Han Ping. "Getting Texture Eigenvalues in Classification and Identification of Stored-Product Pests." Computer Engineering and Applications 39.1(2003):218-219.

[7] Zhang Hongmei. "A Classifying System of Stored-product Pests Based on Support Vector Machines." Computer Engineering and Applications 41.9(2005):216-218.

[8] Otsu, N., "A Threshold Selection Method from Gray-Level Histograms," IEEE Transactions on Systems, Man, and Cybernetics, Vol. 9, No. 1, 1979, pp. 62-66.

[9] Haralick R M, Shapiro L G. "Computer and Robot Vision: Volume I[J] ." IEEE Robotics \& Automation Magazine, 1992, 18(2):121-122. 